\title{
BMJ Open Understanding facilitators and barriers to follow-up after abnormal cervical cancer screening examination among women living in remote areas of Romania: a qualitative study protocol
}

\author{
Linda Nyanchoka (10 , ${ }^{1}$ Andreea Damian, ${ }^{2}$ Mari Nygård ${ }^{1}$
}

To cite: Nyanchoka L,

Damian A, Nygård M. Understanding facilitators and barriers to follow-up after abnormal cervical cancer screening examination among women living in remote areas of Romania: a qualitative study protocol. BMJ Open 2022;12:e053954. doi:10.1136/ bmjopen-2021-053954

- Prepublication history and additional supplemental material for this paper are available online. To view these files, please visit the journal online (http://dx.doi.org/10.1136/ bmjopen-2021-053954).

Received 31 May 2021 Accepted 04 February 2022

Check for updates

(C) Author(s) (or their employer(s)) 2022. Re-use permitted under CC BY-NC. No commercial re-use. See rights and permissions. Published by BMJ.

${ }^{1}$ Department of Research, Cancer Registry of Norway, Oslo, Norway

${ }^{2}$ Institute of Oncology "Prof. Dr. Ion Chiricuță" Cluj-Napoca (IOCN), Cluj-Napoca, Romania

Correspondence to

Ms Linda Nyanchoka;

Inyanchoka@gmail.com

\section{ABSTRACT}

Introduction In 2020 in Europe, Romania had the second highest incidence and mortality rates of cervical cancer, after Montenegro. To address cervical cancer in the country, the Romanian government established a national cervical cancer screening programme in 2012 The programme provides free testing as well as free treatment in the event of cervical precancer diagnosis for women 25-64 years old with health insurance who are referred from a programme-registered general practitioner. Participation in screening, retesting and follow-up for detected precancerous lesions is suboptimal, thus reducing the overall effectiveness of the programme. Aim The overall aim of the study is to examine facilitators and barriers to cervical cancer screening follow-up after an abnormal cervical cancer screening examination among underserved women living in remote areas of Romania. We have the following specific research questions: (1) what are the knowledge, perspectives and experiences of women living in remote areas of Romania with cervical cancer screening and (2) what are the knowledge, perspectives and experiences of women living in remote areas of Romania with follow-up information and services after abnormal cervical cancer screening results.

Method We will conduct an exploratory qualitative study using semi-structured interviews. Data analysis will be based on the thematic analysis outlined by Braun and Clarke. We will use QSR International's NVivo V.12 as the qualitative data analysis software for both data management and analysis.

Ethics and dissemination Study findings will inform recommendations for the Romania national policy for the cervical cancer screening programme, with a particular focus on underserved women living in remote areas with limited access to healthcare services. They will also be disseminated to relevant conferences and meetings. Ethics approval was obtained from Romania (Ref. 199/1501.2021 application no. 661/15.01.2021) and Norway (Ref. 12929853).

\section{BACKGROUND}

Cervical cancer is one of the most common cancers in women after breast, colorectal and lung cancer. ${ }^{1}$ Cervical cancer represents
Strengths and limitations of this study

- This study protocol provides guidance on how to design qualitative research in remote areas to improve cervical cancer screening, ultimately supporting the WHO efforts to eliminate cervical cancer globally.

- The qualitative nature of this study provides an indepth understanding of the knowledge, perceptions and experiences of women living in remote areas with follow-up information and services after an $a b$ normal cervical cancer screening examination.

- This study includes feedback and insight from women attending cervical screening as well as community nurses involved in conducting cervical screening, particularly the development of the interview guide to provide suggestions on developing questions that are important and relevant to them.

- This study is limited to the experiences of women living in remote communities located in two counties, Dolj and Gorj, in Romania.

a major public health problem for large numbers of women globally. ${ }^{1}$ Persistent infection with high-risk human papillomavirus (HPV) is the main cause of cervical cancer. This situation has resulted in the development of prophylactic vaccines to prevent HPV infection and HPV assays that detect nucleic acids of the virus to be used in organised cervical cancer screening programmes. ${ }^{2}$ This development has led to global efforts such as WHO launching a global initiative in 2018 to scale up vaccination and early detection followed by treatment to eliminate cervical cancer as a public health problem during the 21st century. ${ }^{3}$

Despite advances in technology for cervical cancer control, women in low/ and middle-income countries (LMICs) are highly vulnerable to cervical cancer, with about $90 \%$ of cases and deaths from cervical cancer occurring in LMICs. ${ }^{34}$ Cervical cancer 
screening tests such as the Papanicolaou (Pap) and HPV DNA tests are effective ways to detect cervical cancer. ${ }^{35}$ Combined programmes of HPV vaccination and cervical cancer screening have significantly reduced the burden of cervical cancer primarily in high-income countries. However, the burden remains greater in LMICs because of no vigorous mass vaccination, limited screening tests and few treatment facilities. ${ }^{356}$

Failure to pursue follow-up after an abnormal cervical cancer screening could lead to cervical cancer, yet little is known about adherence to recommended follow-up after an abnormal cervical cancer screening result. ${ }^{7}$ Evidence for some of the reasons that contribute to failure to follow-up among women after an abnormal cervical cancer screening result include lack of guidelineadherent clinical management, appropriate care and breakdowns at the provider, patient and/or system levels of care for women with abnormal results. ${ }^{7}$

Romania has the highest incidence and mortality rates of cervical cancer: 22.6 and 9.6 per 100000 women, respectively. ${ }^{8}$ Current estimates indicate that every year in Romania, 3308 women receive a diagnosis of cervical cancer and 1743 die from the disease. ${ }^{9}$ The Romanian government established a national cervical cancer screening programme in 2012. The programme provides free testing as well as free treatment in the event of a cervical cancer precancer diagnosis for all women 25-64 years old when they are referred from a programmeregistered general practitioner. However, retesting and follow-up of detected precancerous lesions are covered for only women with health insurance. Despite the initiation of the national cervical cancer screening programme, participation is low, especially among minority Roma women and other ethnic groups. ${ }^{10}$ One study of Roma women's (non)participation in cervical cancer screening in Romania found major differences in perspectives on screening between users and providers. The study recommended that to improve attendance, all women needed to be involved in the planning, mobilisation, implementation and evaluation of the programme to build trust between those offering screening and the potential participants. ${ }^{11}$

With a goal to reduce the incidence of and mortality from cervical cancer in Romania, a European-funded project was created: 'Strengthening at National Level the Capacity of the Romanian Health Sector to implement organized screening for cancers amenable to costeffective early detection', also known as CEDICROM 2 (2019-2021). This project aims to improve the quality assurance of integrated preventive and curative medical services related to cervical cancer prevention among the disadvantaged population at risk living in communities in remote areas in Romania. It is a follow-up of CEDICROM 1 (2014-2017), which aimed to strengthen the national capacity to implement well-organised and sustainable screening programmes for cervical and breast cancer. One of the key learning points from CEDICROM 1 was the suboptimal follow-up care services provided to women with abnormal cervical cancer screening results in the programme.

Finding cervical cancer often starts with an abnormal HPV test or Pap smear result. This will lead to further tests, which can lead to a diagnosis of cervical cancer or precancer. All adult women should undergo periodic cervical cancer screening, which aims to detect precancerous lesions, that is, abnormalities in cells of the cervix. When found, precancerous lesions must be followed up and treated. Therefore, follow-up abnormal cervical cancer screening is of key importance and can save lives. Our overall aim is to examine facilitators and barriers to cervical cancer screening follow-up among women living in remote areas of Romania. We have the following specific research questions: (1) what are the knowledge, perspectives and experiences of women living in remote areas of Romania with cervical cancer screening and (2) what are the knowledge, perspectives and experiences of women living in remote areas of Romania with follow-up information and services after abnormal cervical cancer screening results.

\section{METHODS AND ANALYSIS}

\section{Qualitative study design}

We will conduct an exploratory qualitative study using semi-structured interviews. This research method will provide opportunities to explore women's knowledge, perspectives and experiences related to cervical cancer screening testing and follow-up care. ${ }^{12-14}$

\section{Study sample and recruitment}

We plan for data collection between July and January 2022. We will use purposive sampling to ensure that the perspectives of women who participated in the cervical cancer screening programme will be included. Purposive sampling is widely used in qualitative research to identify and select information-rich cases. ${ }^{15} 16$ The study sample will recruit women $\geq 25$ years old who participated CEDICROM 1 or 2, had an abnormal Pap smear or positive HPV test result and were required to receive follow-up care. Study participants will be identified in the cervical cancer screening register database of CEDICROM 1 and 2 , which has the contact list of women who attended the cervical cancer screening programme.

The main inclusion criteria for the study are as follows:

- Adult women $\geq 25$ years old.

- Women who participated in the CEDICROM 1 and 2 cervical cancer screening programme who had an abnormal Pap smear or a positive HPV test result and were required to receive follow-up care.

The sample size for qualitative studies usually depends on the point when data saturation is reached (ie, the point when new data does not add to a better understanding of the studied phenomenon but rather repeats what was previously expressed ${ }^{17}$ ). Considering that the point of saturation cannot be specified in advance, we planned to conduct between 20 and 40 interviews according to 
usual points of data saturation reported in qualitative studies. ${ }^{18}$ If we do not achieve saturation based on participant responses and recurring themes, we will increase the number of interviews accordingly. We will determine data saturation during data collection, when new discoveries from the interviews do not add further insights. ${ }^{19} 20$

\section{Data collection and recording}

The interview guide will focus on the following components. The goal is to have open-ended questions and only probe if needed to allow the participants to express their experiences with receiving follow-up after an abnormal cervical cancer screening result and lack of follow-up care.

- Participant background information.

- Cervical cancer screening and abnormal result information experience and test results.

- Women's experiences with follow-up information and services after an abnormal cervical cancer screening result.

- Perceived needs and barriers to obtain medical follow-up care after an abnormal cervical cancer screening result.

- Future recommendations to improve the cervical cancer screening experience.

We will conduct all interviews in Romanian, using a local researcher who speaks Romanian. Additionally, the local researcher will follow the project and ensure scientific rigour during fieldwork by routine meetings with the main researcher $(\mathrm{LN})$. The interviews will be conducted, recorded and transcribed verbatim in Romanian, then translated to English by using a professional translation and transcription service. The data collection tools are all included in the supplementary material: the patient information sheet (online supplemental appendix 1), participant consent form (online supplemental appendix 2) and semi-structured interview guide (online supplemental appendix 3). These items were all translated to Romanian.

\section{Data collection and modifications due to COVID-19}

With consideration of the current COVID-19 pandemic, we plan to conduct data collection in two phases to avoid gathering a large number of study participants in one area and spread out the invitations to potential participants. This process will also require more travel to the study locations over the data collection period (July-January 2022). Both phases will target women from Gorj and Dolj counties in the southwest region of Romania. Because of time considerations, COVID-19 and the geographic spread of Romania, we will limit our data collection to Gorj and Dolj counties (including several cities), which will provide us with experiences from women in only these locations and therefore will not be representative of the other counties in Romania. We are also limited to locations that implemented CEDICROM 1 and 2 and therefore guaranteed that women had cervical cancer screening.
The main modification of this study is limiting the data collection to semi-structured interviews only versus semi-structured interviews and focus groups. The change to the data collection methodology regarding semi-structured interviews aims to limit the gathering of more than two people at a time to ensure COVID-19 preventive measures (ie, social distancing up to $1 \mathrm{~m}$, use of sanitiser and wearing a mask during the interview process). Additionally, we wanted to include more counties, but because of the restrictions, we modified our focus to ensure access to and feasibility with the two counties because we have existing contacts and an established network from the overall CEDICROM 2 programme. This situation will limit our study focus to women's experiences in Dolj and Gorj versus other counties in Romania.

Also, because of travel restrictions, the main researcher (LN) may not be physically present during the interviews to assist the local researcher in Romania who is responsible for data collection. To ensure adequate involvement by the main researcher, $\mathrm{LN}$ will be involved in the interview process via remote access if she is unable to travel and otherwise in person when possible. This situation will allow the main researcher to observe the conduct of the interviews and participant reactions that are important to contextualise and interpret the study findings. All data collected in the field will be securely stored by using password protection and maintained by the main researcher (LN). The data will be stored both in Romania by the local researcher and in Norway, at the Cancer Registry of Norway, by the main researcher (LN).

\section{Ensuring study quality}

To further ensure rigour and trustworthiness, this study will be guided by Guba and Lincoln's concepts for defining and investigating quality in qualitative research that can be considered parallel to quantitative research concepts of validity and reliability. ${ }^{192122}$ The concepts include credibility, transferability, dependability, confirmability, audit trails and reflectivity. The concepts are inter-related, and thinking through them from the onset and incorporating them in a study will improve the study rigour. This process will be detailed further on how we will apply them in the main manuscript with study findings.

Regarding addressing the sensitivity of the topic and the potential stigma it carries for the women, the interviewer(s) will be cognizant of the participant(s) emotional responses that can arise due to the topic and great stigma connected to women who have an abnormal Pap smear and/or HPV-positive test result. The interviewer(s) will discuss this beforehand and will immediately remind the interviewee that they can stop the interview at any time. In addition, if the women would like to continue, the interviewer(s) will provide ample time for them to feel at ease and only continue if they allow it. We will also ensure participant privacy by providing a quiet, well-ventilated room for interviewing. 


\section{Data analysis}

Our approach is based on the thematic analysis outlined by Braun and Clarke. ${ }^{23}$ The steps include (1) transcription and checking transcripts with recordings for accuracy; (2) open coding from interview responses performed by at least two researchers independently; (3) agreement of initial codes discussed among the researchers and an initial codebook developed; (4) developing the code structure used for analysing the remaining responses with openness that include new codes and refining existing ones; and (5) themes and subthemes identified from the final code structure and their relationships presented. ${ }^{23}$ The initial coding framework for our analysis will be inductively derived from the data. In this sense, our approach will include a bottom-up development of analytic categories and themes. We will use QSR International's NVivo V.12 as the qualitative data analysis software for both data management and analysis.

\section{Strengths and limitations}

The qualitative study findings will provide an overview of facilitating factors and barriers to pursue follow-up after an abnormal cervical cancer screening test result. This is the first study in Romania to investigate follow-up after cervical cancer screening; therefore, it provides an opportunity for future research to better understand women's needs and how to improve healthcare services. One main limitation of this study is the current COVID-19 pandemic that has affected the communities where we planned to conduct our study. Hence, we have modified the study to the best of our capacity to ensure safety precautionary measures according to the Romanian government.

\section{Patient/public involvement}

This study will include women attending cervical cancer screening and community nurses involved in providing cervical cancer screening feedback and insight into the study development. A cervical cancer survivor reviewed our interview guide and the appropriateness of the questions planned; additionally, she provided comments on what questions could be most important. Also, two Romanian nurses who participated in cervical cancer screening reviewed the interview guide and provided comments to improve it. Therefore, we focused on both women with cervical cancer screening experience and nurses. The involvement of other patients/the public was limited because of the pandemic and the limited access to the community we planned to conduct our study in.

\section{Ethics}

Permission to conduct the study was obtained before the study from Romania and Norway. In Romania, "Prof. Dr. Ion Chiricuță" Cluj-Napoca on the Ethical Committee of the Oncology Institute approved the project (Ref. 199/1501.2021 application no. 661/15.01.2021). In Norway, a privacy-related impact assessment was conducted to identify and analyse how data privacy might be affected, following institutional Data Protection guidelines (Ref. 12929853). Moreover, an information sheet describing the study in detail and the role of the study participants was developed in English and translated to Romanian. Informed consent will be obtained from all participants before data collection. Anonymity and privacy will be maintained throughout the study. Participants will be allowed to withdraw from the study at any point in the research process, without consequences. The consent form and interview guide were written in English and translated to Romanian. Participants who cannot write and read will be briefed about the aim and nature of the study and asked to provide oral consent to participate in the study.

\section{Twitter Linda Nyanchoka @LindaNyanchoka}

Acknowledgements The authors sincerely thank Margrethe Meo, Trude Andreassen, Gunvor Aasbø, Adriana Melnic, Florian Nicula, Roxana and Jivulescu for their insight, time and input on the development of this study protocol.

Contributors LN conceived the study with feedback from MN and AD; all authors read and approved the final manuscript.

Funding This project is a part of CEDICROM 2. It received grants from EEA/Norway grants (project no.: R0-HEALTH-0002).

Competing interests None declared.

Patient consent for publication Not applicable.

Provenance and peer review Not commissioned; externally peer reviewed.

Supplemental material This content has been supplied by the author(s). It has not been vetted by BMJ Publishing Group Limited (BMJ) and may not have been peer-reviewed. Any opinions or recommendations discussed are solely those of the author(s) and are not endorsed by BMJ. BMJ disclaims all liability and responsibility arising from any reliance placed on the content. Where the content includes any translated material, BMJ does not warrant the accuracy and reliability of the translations (including but not limited to local regulations, clinical guidelines, terminology, drug names and drug dosages), and is not responsible for any error and/or omissions arising from translation and adaptation or otherwise.

Open access This is an open access article distributed in accordance with the Creative Commons Attribution Non Commercial (CC BY-NC 4.0) license, which permits others to distribute, remix, adapt, build upon this work non-commercially, and license their derivative works on different terms, provided the original work is properly cited, appropriate credit is given, any changes made indicated, and the use is non-commercial. See: http://creativecommons.org/licenses/by-nc/4.0/.

ORCID iD

Linda Nyanchoka http://orcid.org/0000-0003-0822-6736

\section{REFERENCES}

1 Arbyn M, Weiderpass E, Bruni L, et al. Estimates of incidence and mortality of cervical cancer in 2018: a worldwide analysis. Lancet Glob Health 2020;8:e191-203.

2 Schlecht NF, Kulaga S, Robitaille J, et al. Persistent human papillomavirus infection as a predictor of cervical intraepithelial neoplasia. JAMA 2001;286:3106-14.

3 Gultekin M, Ramirez PT, Broutet N, et al. World Health organization call for action to eliminate cervical cancer globally. Int J Gynecol Cancer 2020;30:426-7.

4 Richardson LA, Tota J, Franco EL. Optimizing technology for cervical cancer screening in high-resource settings. Expert Rev Obstet Gynecol 2011;6:343-53.

5 World Health Organization. World health assembly adopts global strategy to accelerate cervical cancer elimination. WHO, 2020.

6 Arbyn M, Gultekin M, Morice P, et al. The European response to the who call to eliminate cervical cancer as a public health problem. Int $J$ Cancer 2021;148:277-84.

7 Perkins RB, Adcock R, Benard V, et al. Clinical follow-up practices after cervical cancer screening by co-testing: a population-based study of adherence to U.S. guideline recommendations. Prev Med 2021;153:106770. 
8 WHO. Romania. Available: https://gco.iarc.fr/today/data/factsheets/ populations/642-romania-fact-sheets.pdf

9 Bruni LAG, Serrano B, Mena M. ICO/IARC information centre on HPV and cancer (HPV information centre). In: Human Papillomavirus and Related Diseases in the World Summary Report. 17, 2021.

10 Andreassen T, Melnic A, Figueiredo R, et al. Attendance to cervical cancer screening among Roma and non-Roma women living in north-western region of Romania. Int $J$ Public Health 2018;63:609-19.

11 Andreassen T, Weiderpass E, Nicula F, et al. Controversies about cervical cancer screening: A qualitative study of Roma women's (non)participation in cervical cancer screening in Romania. Soc Sci Med 2017;183:48-55.

12 Côté L, Turgeon J. Appraising qualitative research articles in medicine and medical education. Med Teach 2005;27:71-5.

13 Halcomb EJ, Davidson PM. Is verbatim transcription of interview data always necessary? App/ Nurs Res 2006;19:38-42.

14 McGrath C, Palmgren PJ, Liljedahl M. Twelve tips for conducting qualitative research interviews. Med Teach 2019;41:1002-6.

15 Patton MQ. Qualitative evaluation and research methods. SAGE Publications, 1990.
16 Setia MS. Methodology series module 5: sampling strategies. Indian J Dermatol 2016;61:505.

17 Saunders B, Sim J, Kingstone T, et al. Saturation in qualitative research: exploring its conceptualization and operationalization. Qual Quant 2018:52:1893-907.

18 Nyanchoka L, Tudur-Smith C, Porcher R, et al. Key stakeholders perspectives and experiences with defining, identifying and displaying gaps in health research: a qualitative study protocol. BMJ Open 2019;9:e027926.

19 Shenton AK. Strategies for ensuring trustworthiness in qualitative research projects. Education for Information 2004;22:63-75.

20 Hennink MM, Kaiser BN, Marconi VC. Code saturation versus meaning saturation: how many interviews are enough? Qual Health Res 2017;27:591-608.

21 Glonti K, Hren D. Editors' perspectives on the peer-review process in biomedical journals: protocol for a qualitative study. BMJ Open 2018;8:e020568.

22 Lincoln YS, Guba EG, Pilotta JJ. Naturalistic inquiry. . sage, 1985: 9. 438-9.

23 Braun V, Clarke V. Using thematic analysis in psychology. Qual Res Psychol 2006;3:77-101. 